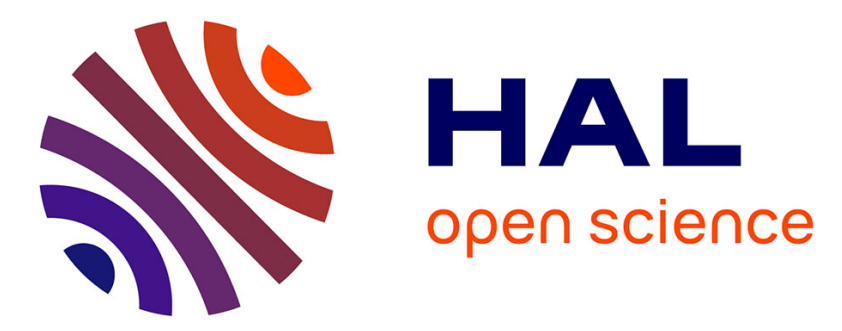

\title{
De la prosopographie à la monographie, ou comment étudier la bourgeoisie parisienne au Moyen Âge \\ Boris Bove
}

\section{To cite this version:}

Boris Bove. De la prosopographie à la monographie, ou comment étudier la bourgeoisie parisienne au Moyen Âge. Le médiéviste et la monographie familiale: sources, méthodes et problématiques, 2003, France. pp.265-282. halshs-00640419

\section{HAL Id: halshs-00640419 \\ https://shs.hal.science/halshs-00640419}

Submitted on 11 Jan 2012

HAL is a multi-disciplinary open access archive for the deposit and dissemination of scientific research documents, whether they are published or not. The documents may come from teaching and research institutions in France or abroad, or from public or private research centers.
L'archive ouverte pluridisciplinaire HAL, est destinée au dépôt et à la diffusion de documents scientifiques de niveau recherche, publiés ou non, émanant des établissements d'enseignement et de recherche français ou étrangers, des laboratoires publics ou privés. 


\section{« DE LA PROSOPOGRAPHIE A LA MONOGRAPHIE, OU COMMENT ETUDIER LA BOURGEOISIE PARISIENNE AU MOYEN ÂGE »}

C'est à dessein que le titre de la présente contribution prend le contre-pied de celui de la séance... et du sens commun, tant il est vrai que l'Histoire, toujours empirique, va plutôt du particulier au général, d'une famille au groupe, de l'analyse à la synthèse, c'est-à-dire de la monographie (traditionnellement qualitative) à la prosopographie (supposée quantitative). Cette évidence se heurte cependant à un obstacle épistémologique dès lors que l'on ne s'intéresse plus à un groupe, c'est-à-dire un ensemble, forcément limité, d'individus en relation entre eux, mais à une catégorie de personnes qui embrasse une large portion de la société : comment, dès lors, toutes les étudier ? et quand bien même y parviendrait-on, aura-ton compris cette catégorie sociale à partir de cette collection de cas ? La monographie peut être le point de départ du premier type d'études, mais plus difficilement du second.

Le problème se pose avec une acuité particulière pour la bourgeoisie de Paris. En effet, la capitale compte, au tournant des $\mathrm{XIII}^{\mathrm{e}}$ et $\mathrm{XIV}^{\mathrm{e}}$ siècles, au moins 200.000 habitants, dont peutêtre 15.000 à 30.000 paient la taille et peuvent, de ce fait, être juridiquement considérés comme «bourgeois de Paris ${ }^{1}$. Cette dimension démographique interdit à elle seule toute entreprise de mise en série systématique de monographies familiales - on hésiterait d'ailleurs à qualifier de «monographique » toute étude d'ensemble de la bourgeoisie parisienne, tant l'objet est énorme à l'échelle des capacités d'appréhension de l'historien. Ce problème épistémologique se complique, dans le cas parisien, d'un problème social, et d'un autre, documentaire. En effet, la bourgeoisie de Paris est hétérogène et réunit dans un même statut juridique de riches marchands et de modestes artisans, avec tous les degrés entre ces deux idéal-types. Enfin, les sources qui renseignent sur la vie des bourgeois sont très abondantes, mais aucune ne les concerne particulièrement (il n'y a pas d'archives familiales, notariales, ni même municipales à Paris avant le $\mathrm{XV}^{\mathrm{e}}$, voire le $\mathrm{XVI}^{\mathrm{e}}$ siècle), si bien que la collecte des informations est sans fin et les données très allusives. En tant qu'objet historique, Paris est un mammouth documentaire dont l'anatomie est très mal connue, faute d'instruments de

\footnotetext{
${ }^{1}$ Le chiffre exact du nombre de contribuables est difficile à établir parce que les rôles de taille de 1292, 1296, 1297, 1298, 1299, 1300 et 1313 ne recensent pas exactement la même population à chaque fois : non seulement le nombre de chefs de feux taxés varie du simple au double d'une année sur l'autre sans qu'aucun accident démographique ne le justifie, mais encore des études de détail montrent que beaucoup sont taillés épisodiquement, comme si les moins riches payaient à tour de rôle. Par conséquent, le nombre de Parisiens qui paient la taille, et peuvent de ce fait être considérés comme bourgeois, excède largement les 15.038 taillables recensés en 1292. Il paraît raisonnable de penser qu'il ne dépasse pas le double.
} 
recherche nombreux et précis. Ces contraintes expliquent que les monographies familiales y soient rares et souvent décevantes ${ }^{2}$. Autant dire que l'on n'est pas près de faire de la micro-histoire à l'italienne à Paris.

L'écueil documentaire est plus visible que l'écueil épistémologique ; c'est probablement pourquoi Raymond Cazelles, dans sa grande synthèse sur Paris de la fin du règne de Philippe Auguste à la mort de Charles $V$, affirme que «l'histoire [des grandes familles bourgeoises ne sera bien connue, avec l'origine de leur fortune et leurs alliances, que lorsque des monographies leur auront été consacrées $\gg^{3}$ et propose ensuite une liste de 6 familles, définies par leur patronyme (les Bourdon, Sarrazin, Arrode, Pisdoe, Gencien et Popin), à étudier en priorité. Pour lui, l'échelle de la monographie familiale s'impose comme une évidence et il suggère une division du travail universitaire par famille (« ce pourrait être l'objet de diplômes et de thèses utiles »), laissant entendre que la synthèse appartiendrait ensuite à un chercheur chevronné - un professeur? Mais alors, pourquoi travailler sur ces 6 familles là, précisément? Et pourquoi sur 6 seulement? Et pourquoi pas sur 60, ou même 600 ? Cet arbitraire - Raymond Cazelles ne propose rien moins que de tailler un steak, au hasard, dans le mammouth - met le doigt sur les contradictions propres au genre monographique, pris entre son souci de connaissance approfondie, systématique, intime de la réalité humaine et, qu'on l'avoue expressément ou non, l'ambition holiste de ses conclusions. Cela revient, au fond, à poser le problème de la pertinence de l'échelle monographique (familiale) dans l'analyse globale de la société ${ }^{4}$.

\footnotetext{
${ }^{2}$ A. Bloch-Michel, «Michel Lallier, bourgeois de Paris (vers 1370-1440)», Position des thèses de l'Ecole des chartes, 1950, p 11-12; Léon-Louis Borelli de Serres, «Les Gencien tués à Mons-en-Puelle (1314)», in Recherches sur divers services publics du XIII ${ }^{e}$ au XVII ${ }^{e}$ siècle, Paris, 1, 1895, p 575-608; D. Bossuat, «Guillaume Sanguin, bourgeois de Paris (1370 ?-1442) », in Position des thèses de l'École des chartes, 1945, p. 59-62 ; J. Délivré, «Un prévôt des marchands [de Paris] au $\mathrm{XV}^{\mathrm{e}}$ siècle : Michel de la Grange », Les amis du vieux Maincy, 1992-93, $\mathrm{n}^{\circ} 14$, p 24-28 et «Un prévôt des marchands du temps de Louis XI : Henry Délivré », B.S.H.P., 105, 1978, p.75-96 ; J. Hoareau-Dodinau, «Laurent de Veulettes, un révolutionnaire de 1358 », Mem. Soc. Droit Pays Bourguignon, 1989, fasc. 46, p 80-94 ; Charles-Victor Langlois, « Geoffroi des Nés, ou de Paris, traducteur et publiciste », in Histoire Littéraire de la France, 35, Paris, 1921, p.324 ; André Lesort, "Gilles de Clamency, bourgeois de Paris et de Rouen, négociant et armateur à la fin du XIV siècle et au début du XV siècle », Bull. historique et philologique du comité des travaux historiques et scientifique, 1958, p 227-236; Philippe Morel, «Une famille de la bourgeoisie parisienne, les Barbette », B.S.H.P., 99-100, 1972-1973, p 3961 ; Charles Selliers, Monographie historique et archéologique d'une région de Paris. Le quartier Barbette, Paris, 1899 ; Anne Terroine et Lucie Fossier, Un bourgeois parisien du XIII ${ }^{e}$ siècle, Geoffroy de Saint-Laurent (1245?-1290), Paris, 1992; Anne Terroine, «Gandoulfe d'Ancelles et les compagnies placentines à Paris (fin XIII ${ }^{\mathrm{e}}$ siècle), Annales d'histoire sociale, 1945, p. 54-71.

${ }^{3}$ Raymond Cazelles, Paris de la fin du règne de Philippe Auguste à la mort de Charles V (Nouvelle Histoire de Paris, t. IV), Paris, 1972, p. 97.

${ }^{4}$ Christian Bromberger, "Monographie », in Dictionnaire de l'ethnologie et de l'anthropologie, dir. Pierre Bonte et Michel Izard, Paris, 1991, p. 485-486 et «Du grand au petit. Variations des échelles et des objets d'analyse dans l'histoire récente de l'ethnologie en France », in Ethnologies en miroir. La France et les pays de langue allemande, dir. Isac Chiva et Utz. Jeggle, Paris, 1987, p. 67-94; Jacques Revel , «micro-analyse et
} 
Il me semble que pour trouver une solution aux trois problèmes évoqués plus haut autrement que par un choix aussi arbitraire que contestable sur le plan épistémologique, il faut renoncer à la monographie traditionnelle, telle que l'entend Raymond Cazelles, et qu'un détour par la prosopographie parait salutaire ${ }^{5}$. Cela ne signifie pas pour autant que l'on ne peut ensuite intégrer la monographie au raisonnement. En fait ces deux démarches, loin de s'opposer, sont complémentaires... à condition de bien les ordonner.

\section{Prosopographie contre monographie}

Qui veut étudier la bourgeoisie parisienne comprend vite pourquoi il ne faut pas commencer par une monographie familiale. L'historiographie parisienne est, à ce titre, caricaturale. Elle est écartelée entre une érudition très pointue, à l'ancienne, telle qu'on la trouve par exemple dans les Mémoires ou les Bulletins de la société de l'histoire de Paris et de l'Île-de-France depuis $1876^{6}$ et des synthèses très générales, celle de Marcel Poëte en 1924 et celle de Raymond Cazelles en 1972 qui mettent en œuvre les exemples tirés de l'érudition ${ }^{7}$. Ces grandes synthèses brillent par leur clarté et la hauteur de leurs vues, mais, outre qu'elles sont sans note, on se demande toujours quelle est la représentativité des cas qui étayent l'argumentation. Par exemple, celui de Geoffroy Cocatrix, qui a été «trésorier des guerres, pourvoyeur et maître des garnisons, collecteur ou receveur de plusieurs subventions, commissaire pour la fausse monnaie, maître et visiteur des ports et passages du royaume, et autres offices » ne manque pas d'être convoqué pour illustrer les carrières des bourgeois au service de l'Etat et les possibilités d'ascension sociale qui en découlent ${ }^{8}$, sans qu'on sache ce qui est exemplaire et ce qui ne l'est pas dans son itinéraire...

construction du social », in Jeux d'échelles. La micro-analyse à l'expérience, dir. Jacques Revel, Paris, 1996, p. 15-36.

${ }^{5}$ C'est l'objet de ma thèse, parue sous le titre Dominer la ville. Prévôts des marchands et échevins parisiens de 1260 à 1350, Paris, 2004. On y trouvera les références érudites et le raisonnement qui mènent aux conclusions évoquées ici.

${ }^{6}$ Outre les références citées n. 2, on trouve dans ces volumes beaucoup d'édition de sources, qui concernent parfois directement la bourgeoisie, mais qui sont rarement interprétées, comme A. Goldmann, «Inventaire de Galeran le Breton et testament de Jeanne de Malaunay, bourgeois de Paris (1299-1311) », B.S.H.P., 1892, p. 163-170 ; Léon Le Grand, «Testament d'une bourgeoise de Paris [Sédile de Laon] », Ibid., 1887, p. 42-47; Henri Martin, «Le testament de Simon Pizdoe », Ibid., 31, 1904, p 33-39... Que l'on ne se méprenne pas : cette production historiographique est remarquable par sa rigueur et les publications des sociétés savantes ont permis de rendre accessible de nombreuses synthèses partielles sur l'histoire de Paris, mais la plupart, portées par la structure des archives, s'attachent plus aux institutions ou aux lieux qu'aux hommes.

${ }^{7}$ Marcel Poëte, Une vie de Cité. Paris de sa naissance à nos jours, t. I, La jeunesse : des origines aux temps modernes, Paris, 1924 ; Raymond Cazelles, Paris..., op. cit.

${ }^{8}$ Marcel Poëte (Une vie de Cité..., p. 449) puise ce tableau directement dans la documentation brute (charte éditée par Henri Frémaux, «La famille d'Étienne Marcel », M.S.H.P., 30, 1903, pi. justif. XIV), pour une fois synthétique, car le personnage - un fils de tavernier parisien qui fit fortune en fournissant les armées de Philippe 
C'est, au fond, toujours la représentativité du cas qui est en cause, et cette question est d'autant plus lancinante à Paris qu'il n'y a pas d'étage intermédiaire, de «monographies de synthèse », entre les études d'érudition et les ouvrages très généraux ${ }^{9}$. La prosopographie, entendue comme la description des caractères externes d'une collection d'individus à partir de caractéristiques observables (nom, carrière, impôt, alliances, culture, etc. ${ }^{10}$, paraît à cet égard doublement rigoureuse. D'abord parce qu'elle porte sur un grand nombre d'individus, ce qui limite la probabilité de recenser seulement des exceptions et élimine la question de la place de l'exemple par rapport à l'ensemble, ensuite parce que l'historien, dans le portrait qu'il fait de ces individus, s'en tient autant que possible aux éléments objectifs, c'est-à-dire répétitifs, ce qui limite la part d'intuition psychologisante qui est souvent à l'œuvre dans la biographie.

La prosopographie est donc «scientifique » en ce sens qu'elle se rapproche des canons épistémologiques de la sociologie, qui elle-même a emprunté les siens aux sciences dures, pour qui il n'y a de science que du général et de l'abstrait - ce qui est extrêmement ennuyeux pour la discipline historique, toujours concrète et particulière, parce que contextualisée ${ }^{11}$. Ce rapprochement avec la sociologie est tardif. Il est moins propre à la méthode elle-même qu'au contexte historiographique des années 1960 qui a conduit les historiens, soucieux de prendre en compte les critiques du sociologue François Simiand (en particulier celle visant «l'idole individuelle ») ${ }^{12}$, à replacer les cas exceptionnels dans les mouvements de masse, et à utiliser cette vieille science auxiliaire utilisée depuis la fin du XIXe siècle par les historiens de l'Antiquité pour appliquer le questionnement sociologique à une documentation préstatistique ${ }^{13}$.

le Bel - est célèbre, mais sa carrière touffue. Raymond Cazelles esquisse ensuite la monographie de cet homme hors du commun, mais cette dernière reste à faire (Paris..., p. 108).

${ }^{9}$ La structure des sources a plutôt orienté les historiens de Paris vers des synthèses institutionnelles ou topographiques, plus rarement vers des synthèses sociales. Dans ce dernier cas, elles font le plus souvent le grand écart entre les agrégats juridiques et conceptuels médiévaux et des cas glanés au fil des dépouillements (voir par exemple J.-M. Archer, Working women in thirteenth-century Paris, thèse de l'Université d'Arizona, Ann Arbor, Michigan, 1995 ; Gustave Fagniez, Étude s sur l'industrie et la classe industrielle à Paris aux XIII ${ }^{e}$ et XIV siècles, Paris, 1877 ; Bronislaw Geremek, Le salariat dans l'artisanat parisien aux XIII ${ }^{e}-X V^{e}$ siècles, Paris, 1962 et Les marginaux parisiens aux XIV et XV siècles, Paris, 1976 ; Anne Terroine, Recherches sur la bourgeoisie parisienne au XIII siècle, thèse de l'Ecole des chartes, 1939).

${ }^{10}$ Jean-Philippe Genet, «Introduction », in Prosopographie et genèse de l'État moderne, éd. Françoise Autrand, Paris, 1985, p. 10.

${ }^{11}$ Antoine Prost, Douze leçons sur l'histoire, Paris, 1996, p. 189-197.

${ }^{12}$ François Simiand, «Méthode historique et sciences sociales. Etude critique d'après les ouvrages récents de M. Lacombe et de M. Seignobos », Revue de synthèse historique, VI, 17, 1903, p. 154-155.

${ }^{13}$ La prosopographie consiste au départ à identifier toutes les personnes citées par les auteurs antiques - c'est-àdire les notables -, puis à en tirer des notices qui viennent grossir un catalogue biographique. C'est seulement dans l'après guerre que les historiens se sont appuyés sur ces identifications pour repenser, dans une perspective sociale, les catégories juridiques de «peuple », « sénat », « ordre équestre » qui servaient de depuis l'Antiquité à appréhender l'histoire romaine. (Claude Nicolet et André Chastagnol, «Prosopographie et histoire sociale : Rome et l'Italie à l'époque républicaine », Annales ESC, 1970, 25, p. 1209-1235). 
La pertinence scientifique de la méthode étant posée, reste alors à l'appliquer à la bourgeoisie de Paris. Il s'agit de réussir une analyse quantitative de cette catégorie sociale populeuse dans des temps raisonnables, c'est-à-dire la durée d'une thèse nouveau régime - on notera au passage que les contraintes inhérentes au cadre institutionnel dans lequel se réalise la recherche sont des données rarement évoquées alors qu'elles conditionnent pourtant une grande partie des ambitions du travail : ce pourrait bien être, pourtant, un objet d'interrogation épistémologique aussi pertinent que celui de la monographie familiale... La solution réside dans le choix d'un indicateur, sans lequel il n'y a pas d'histoire quantitative. Il s'est porté sur les personnes qui ont accédé au pouvoir municipal, car un topos médiéval que l'on doit à Philippe de Beaumanoir veut que les membres des échevinages urbains du royaume se recrutent toujours au sein de l'élite bourgeoise locale, oligarchique et ploutocratique ${ }^{14}$. Cet indicateur est pertinent à plusieurs titres : les individus ainsi recensés ont tous un point commun (l'exercice de la magistrature urbaine) qui préfigure une certaine homogénéité sociale ; ces personnes appartiennent à l'élite, ce qui est un gage de visibilité archivistique ; ce choix limite l'étude à un nombre réduit de cas; enfin, il s'agit d'une catégorie sociale répandue dans toute l'Europe, ce qui est le gage de perspectives comparatistes, et en particulier de la confrontation du cas parisien avec l'idéal-type du patricien médiéval, tyran local qui cumule pouvoirs économique et politique. Ce dernier point est une garantie supplémentaire de scientificité, puisque les cas étudiés trouveront non seulement leur place dans la société parisienne, mais encore dans la société urbaine européenne.

Sachant que le collège municipal est composé d'un prévôt des marchands assisté de quatre échevins, élus pour un an si on en croit les statuts de la municipalité au $\mathrm{XV}^{\mathrm{e}}$ siècle ${ }^{15}$, et que la première municipalité a duré 122 ans (1260-1382), on peut poser l'hypothèse qu'il y a eu 610 mandats $(5 \times 122)$ sur cette période. Toutefois l'expérience prouve, lorsque les listes des magistrats sont moins lacunaires, que les échevins restent en poste 7 ans en moyenne, ce qui fait qu'il n'y a probablement eu que 87 magistrats effectifs environ (610/7). La recherche a porté sur un nombre d'individus plus réduit encore, moins à cause des lacunes de la liste des échevins, telle que j'ai pu la reconstituer à partir de sources non municipales et qui aboutit à un recensement empirique de 65 magistrats, que par les contraintes documentaires et scolaires évoquées plus haut. Elles m'ont contraint à arrêter l'étude en 1350. Le corpus étudié comprend au bout du compte 47 magistrats municipaux, qui appartiennent eux mêmes à 30

\footnotetext{
${ }^{14}$ Philippe de Beaumanoir, Coutumes de Beauvaisis, éd. Am. Salmon, Paris, 1900, § 1520, 1522, 1525.

${ }^{15}$ La première municipalité a été dissoute à la suite de la révolte des Maillotins en 1382 ce qui a conduit à la dispersion de ses archives. On peut supposer que celle qui est rétablie en 1412 obéissait aux mêmes règles.
} 
familles. Arrêter l'étude à la fin du règne de Philippe VI permettait de laisser de côté le problème de la révolte d'Étienne Marcel, qui est un énorme dossier, aussi historique qu'historiographique, pour se concentrer sur la période antérieure à la crise. L'ambition quantitative est donc finalement limitée, d'où la question :

\section{À quel seuil quantitatif l'histoire cesse-t-elle d'être scientifique?}

Réduire la population de référence à 47 individus nuit-il à la mesure des phénomène que l'on souhaite mesurer à partir d'elle ? Il semble que non, pour deux raisons.

D'abord parce que cet échantillon est suffisant si on sait ce qu'il représente. En l'occurrence il s'agit de plus de la moitié des magistrats de la période, ce qui rend nul le risque de ne recenser que des cas marginaux. Il faut seulement garder à l'esprit que ces personnes ont vécu à une époque où la bourgeoisie parisienne est à son apogée. En outre, les comptages ont du sens dès lors que l'on ne cherche pas de subtilités statistiques. Ce panel est une efficace pierre de touche pour cerner le comportement de la bourgeoisie échevinale si l'on s'en tient à une mesure binaire (oui / non) ou par grande proportion (un quart, deux tiers) des phénomènes. Ainsi on peut vérifier le point de vue de Philippe de Beaumanoir en se demandant si des liens familiaux unissent effectivement les prévôts des marchands et d'échevins de Paris. La réponse est sans ambiguïtés : 30 sur 47 ont un lien de parenté direct ou proche (père, fils, oncle, neveu, cousin germain) et seuls 5 sur 47 n'ont apparemment aucun lien de parenté (même éloignée) ou d'amitié, ce qui est très peu compte tenu de l'existence inévitable de cas non renseignés. Le test statistique donne donc raison au bailli de saint Louis et permet d'affirmer que l'échevinage se recrute dans une nébuleuse de lignages apparentés, ce qui est le gage d'une forte homogénéité sociale.

Ensuite cet échantillon de 47 prévôts des marchands et échevins est pertinent statistiquement parce qu'il s'agit d'une population souche de laquelle on peut tirer des populations dérivées qui comprennent plus d'individus. Par exemple, si l'on s'intéresse à la façon dont ces bourgeois placent leur argent dans les rentes, on raisonne sur un corpus de 204 rentes qui sont passées entre leurs mains, dont $70 \%$ portent sur des immeubles intra muros et sont de faible valeur ( 3 £p. en moyenne), tandis que $16 \%$ portent sur des institutions comme le trésor royal et sont de forte valeur (40 £p en moyenne). Ces statistiques grossières suffisent à mettre en évident des stratégies financières, entre petites rentes spéculatives, parce que susceptibles d'hypothéquer les maisons sur lesquelles elles portent, et gros placements de sûreté gagés sur le Trésor, destinés aux filles à marier ou aux orphelins mineurs. 
Mais il est une population dérivée qui nous intéresse particulièrement ici, c'est celle des 30 familles dans lesquelles se recrutent les magistrats de la ville. Par «famille » j'entends le plus souvent l'ensemble des personnes rassemblées dans le tableau de filiation de l'échevin, mais aussi parfois l'ensemble des personnes portant le même patronyme, car il est souvent difficile d'assigner sa place à chacun dans l'arbre généalogique. Si le nombre d'individus statistique se réduit, ce changement d'échelle met cependant en œuvre des centaines - peutêtre un millier ? - de personnes, soit beaucoup plus que le panel de référence ${ }^{16}$. Le passage de l'échelle individuelle à l'échelle familiale est nécessaire à chaque fois que le questionnement historique est pris dans une impasse, qu'elle soit méthodologique ou documentaire. Ainsi, ce changement d'échelle s'avère indispensable lorsqu'on s'interroge sur la notoriété des échevins : il serait non seulement malaisé, faute d'indicateur évident, mais surtout absurde de mesurer leur notoriété à l'échelle individuelle, puisque le première dimension de leur notabilité c'est leur patronyme, qu'ils partagent avec tous les membres de leur famille. Il paraît donc plus pertinent de mesurer la notoriété à l'échelle du lignage, en prenant comme critère, par exemple, la trace laissée par ce nom dans la toponymie urbaine. Or il s'avère que 14 familles sur 30 ont laissé leur patronyme à une rue, ce qui est considérable compte tenu du nombre d'habitants et de la concentration d'élites que l'on trouve dans la capitale. L'impasse peut être aussi documentaire lorsque le test statistique conduit à croiser une liste de magistrats qui s'étale sur 90 ans et des sources qui portent sur quelques dizaines d'années, parfois moins : le résultat n'est pas significatif, puisque une partie des individus du panel, trop jeunes ou trop vieux par rapport à la source, ne peuvent y figurer. Ainsi, mesurer la pénétration des bourgeois sur le marché curial n'a pas grand sens à l'échelle des échevins (10 sur 47 fournissent le roi, soit moins d'un quart), car les comptes royaux couvrent surtout la période du règne de Philippe le Bel (1285-1314); cette mesure est plus pertinente à l'échelle des familles dont la longévité approche le siècle : on constate alors que 19 familles échevinales sur 30, soit les deux tiers, fournissent le roi et sa famille, ce qui donne une autre appréhension de la réalité. L'échelle familiale est donc pertinente, mais n'apparaît que dans un second temps : cette échelle de substitution n'a de sens, ici, que si elle dérive du panel de référence, individuel, qui donne son unité institutionnelle, puis sociale, à la population.

\footnotetext{
${ }^{16}$ Le nombre n'est pas assuré, car il aurait fallu identifier chaque membre du lignage parmi les nombreux homonymes que présente une documentation dénuée de toute source de type généalogique ou même familial. Cette identification aurait nécessité une recherche approfondie pour chaque cas, ce qui était matériellement impossible.
} 


\section{Efficacité de la prosopographie}

La prosopographie a fait la preuve de son efficacité en permettant de réviser un certain nombre d'idées reçues qui courent dans l'historiographie parisienne, parmi lesquelles on retiendra la place majeure de l'ascension sociale dans la réussite de la bourgeoisie parisienne : Marcel Poëte et Raymond Cazelles ne manquent pas de souligner la percée fulgurante de certains individus, tels Geoffroy Cocatrix, à la faveur de la croissance économique du XIII ${ }^{\mathrm{e}}$ siècle. Ils citent les mêmes exemples, toujours les plus visibles et les plus pittoresques, comme celui de Jean Poinlasne qui «gagnait sa vie en colportant, à travers les rues, de la viande dans un récipient qu'il prit soin - parvenu plus tard à la richesse, de faire enchâsser, comme souvenir de ses humbles débuts, dans une monture d'or et d'argent ». L'anecdote est d'autant plus édifiante qu'elle est issue d'un sermon de Pierre de Limoges ${ }^{17}$. On notera encore que le défaut de «monographies de synthèse » conduit à isoler des exemples dans la documentation brute, sans pouvoir les mettre dans leur contexte social. Ce topos ne résiste pas au test statistique : seuls 6 échevins sur 47 sont des hommes dont le nom était inconnu à Paris avant qu'ils le mettent à l'honneur, et, parmi eux, 3 sont des notables de province récemment installés dans la capitale. Autant dire que les hommes nouveaux, si leur existence est indubitable, sont des cas marginaux au sommet de la société bourgeoise. L'ascension sociale au sein de la bourgeoisie conduisant, selon la même idée reçue, tout droit à la noblesse ${ }^{18}$, il convenait aussi d'interroger la population de référence sur cette question : 0 anoblissement taisible et seulement 5 familles sur 30 anoblies par lettre, sans payer, sans renoncer au titre de «bourgeois de Paris », sans épouser de «demoiselles », et le plus souvent sans porter de titre nobiliaire...

La prosopographie a aussi une efficacité rhétorique, pour peu qu'on sache l'exploiter dans le discours. L'usage littéraire des cas que l'on peut en tirer est une dimension de l'efficacité de la prosopographie dont on n'ose à peine parler. Il rejoint en effet, avec le problème des conditions matérielles et institutionnelles de la recherche, le tabou de la non-scientificité. Evoquer cette question, c'est admettre que l'Histoire est un discours qui obéit à des règles

\footnotetext{
${ }^{17}$ Marcel Poëte, Une vie de Cité..., p. 222, repris par Raymond Cazelles, Etienne Marcel, champion de l'unité française, Paris, 1984, p. 19. Aucun ne cite sa source mais il s'agit d'une paraphrase de Pierre de Limoges : «Ce bourgeois (...) avait eu des débuts forts pénibles. Il était tellement pauvre, qu'il ne possédait au monde qu'un grand plat, dans lequel il allait porter de la viande en ville pour gagner son pain. Quand il fut devenu un des plus riches personnages de Paris (...) il fit enchâsser dans l'or et l'argent son vieux plat et il le tenait pour aussi précieux qu'une relique. Aussi, à toutes les bonnes fêtes, avait-il soin de se le faire présenter, pour évoquer la mémoire de ses jours de misère » (Albert Lecoy de la Marche, Le rire du prédicateur. Récits facétieux du Moyen $\hat{A} g e$, présentation et notes Jacques Berlioz, Belgique, 1992, p. 95-96, 173-174).

${ }^{18}$ Marcel Poëte, Une vie de Cité..., p. 443, 454 et Raymond Cazelles, Paris..., p. 109-112.
} 
rhétoriques, qui n'ont d'autre légitimité qu'esthétique ${ }^{19}$. L'usage du cas que j'évoque ici n'est pas le même que celui que l'on peut dénoncer dans les synthèses sur l'histoire de Paris, qui montent en épingle un exemple bien servi par la documentation : cet exemple sert alors effectivement à fonder le raisonnement, par conséquent il a un usage « scientifique », même si on peut en contester la pertinence, tandis que le «beau cas » qui m'occupe maintenant n'a d'autre finalité qu'ornementale. L'instrumentalisation littéraire du cas d'espèce passe par deux procédés différents et complémentaires : d'une part la description approfondie de certains exemples, avec citation à l'appui, qui crée un effet de réel, et d'autre part, dans un cadre prosopographique, le souci de corriger l'inégale épaisseur des cas en tâchant de les mettre tous à l'œuvre dans le discours historique, plutôt que de s'appuyer toujours sur un seul exemple, qui serait pourtant assez documenté pour illustrer toutes les étapes du raisonnement. Cela conduit paradoxalement à une sur-représentation relative des cas les moins documentés pour mieux convaincre : ainsi le nombre d'actes dans lesquels chacun des 47 magistrats municipaux est mentionné n'est pas proportionnel avec celui de leurs occurrence dans le livre (graphique 1). Aucun de ces deux procédés n'a d'incidence sur la rigueur et la justesse des conclusions; en revanche ils ont un effet indirect et quasi subliminal sur le sentiment de conviction du lecteur, toujours sensible à la dimension imaginative de la compréhension et à la multiplication astucieusement répartie des exemples... Mais cela nous éloigne des questions strictement familiales.

Si la prosopographie des échevins ou de leur famille permet indubitablement de cerner avec plus de justesse les phénomènes, elle ne permet pas de tous les appréhender, qu'elle butte sur les lacunes des sources ou sur des documents irréductibles à la statistique.

\section{Retrouver la monographie, familiale ou non}

La monographie, familiale ou non, vient d'autant mieux suppléer les carences de la prosopographie qu'elle porte sur des individus ou des familles qui appartiennent à une catégorie sociale (ici, un groupe) dont l'homogénéité est avérée. La monographie s'impose alors naturellement pour exploiter les aubaines documentaires : il s'agit d'exploiter un cas anormalement documenté pour répondre à des interrogations que la méthode statistique est impuissante à résoudre, parce que les cas ne sont jamais renseignés sur ce point.

Ainsi, on peut avoir l'intuition, à la lecture des rôles d'imposition, que la famille des échevins a une structure polynucléaire : les collecteurs de la taille mentionnent souvent des contribuables portant le même patronyme à la suite les uns des autres, ou à proximité, dans la

\footnotetext{
${ }^{19}$ Antoine Prost, Douze leçons..., 263-277.
} 
même rue. Ainsi, 23 des 26 échevins cités dans les tailles ont un voisin qui porte le même nom. Cette répartition en chapelet le long des rues de Paris ne signifie pas toutefois que ces personnes vivent sous le même toit : ils habitent peut-être des maisons mitoyennes (ce qui n'est même pas assuré, car ils peuvent être séparés par un voisin non imposé ou un terrain vague) et ils sont peut-être parents, mais il est rare que les liens familiaux soient précisés par les percepteurs, tandis que les généalogies de ces familles sont trop incomplètes pour qu'on puisse trancher cette question.

C'est là qu'une des rares monographies familiales portant sur la bourgeoisie parisienne, celle des Marcel, est d'un grand secours. Cet hapax historiographique s'explique par la passion que les historiens de la $\mathrm{III}^{\mathrm{e}}$ République vouèrent à Étienne Marcel, le prévôt des marchands qui prit la tête des premiers états généraux, en 1355-1357, puis de la révolte qui s'en suivit en 1358. Ils virent dans l'événement une annonce quasi prophétique de la Révolution de 1789. Ces mauvaises raisons ont eu pour effet positif de mettre en lumière toute la documentation disponible sur la famille, dont l'arbre généalogique est parfaitement maîtrisé ${ }^{20}$. Cette aubaine historiographique coïncide, par chance, avec une aubaine documentaire puisque cette famille est aussi riche en capital qu'en enfants et semble au faîte de sa puissance vers 1300, à l'époque où les sources sont les plus abondantes. Ces heureux hasards permettent de résoudre les interrogations laissées en suspens par la méthode statistique.

La relecture des tailles à la lumière d'une généalogie ferme permet d'affirmer que la structure en chapelet est bien celle d'une même famille (tableau 1), éclatée entre cinq pôles de résidence différents, avec un ancrage principal dans la Draperie. On constate aussi que le père de famille, Pierre III Marcel le Vieux, rassemble autour de lui une bonne partie de ses fils, voire de ses petits-fils, adultes et mariés, mais que ce groupe polynucléaire ne se compose pas des mêmes personnes d'une année sur l'autre (il n'y a qu'à suivre les différents lieux de résidence de Jacques $\mathrm{I}^{\mathrm{er}}$, par exemple), non pas qu'ils déménagent tous les ans, mais plutôt qu'ils occupent collectivement plusieurs résidences à la fois dans Paris, ce qui est le signe de la cohésion effective de cette famille réellement polynucléaire, à défaut d'être celui d'une

\footnotetext{
${ }^{20}$ Eugène Déprez, «Les enfants d'Étienne Marcel », B.S.H.P., 1897, p 83-96; Henri Frémaux, «La famille d'Étienne Marcel », M.S.H.P., XXX, 1903, p. 175-242; Georges Huisman, «Les débuts de la dictature d'Étienne Marcel », Bull. de la Bibl. et des trav. hist. de Paris, 1913, p XXX-XLIV ; Léon Le Grand, « La veuve d'Étienne Marcel », B.S.H.P., 1897, p 140-152; Siméon Luce, «La famille d'Étienne Marcel et les créanciers de sa succession », in La France pendant la guerre de Cent Ans, t 1, 1890, p 49-58 ; R. Morel, «Étienne Marcel. Ses envoyés à Compiègne. Sa trahison. Sa mort », in Procès verbaux de la Soc. hist. de Compiègne, 1892, p. 2945 ; Jules Tessier, «La mort d'Étienne Marcel», Revue de l'enseign. secondaire et de l'enseign. sup., 1886, p. 135-156 et Étienne Marcel, Paris, 1888. De nombreuses biographies lui ont été aussi consacrées, parfaitement critiquées et exploitées par la dernière en date, celle de Raymond Cazelles, Etienne Marcel...
} 
résidence sous le même toit, très improbable. La confrontation des sources fiscales avec celles des seigneurs urbains - en l'occurrence Saint-Éloi - permet de constater que le seul Marcel que mentionne le censier dans la Draperie est Pierre III, qui y acquiert progressivement une, puis trois, puis cinq parcelles contiguës, qui forment son hôtel (carte 1). La stabilité de Pierre le Vieux et la mobilité de ses enfants s'explique donc par le fait qu'il les loge dans des maisons qui lui appartiennent, et l'explosion du groupe à la mort du pater familias est la conséquence logique du partage de l'héritage immobilier. C'est donc l'argent, l'héritage à venir, qui cimente le groupe familial autour du père, tant qu'il est en vie.

Ce cas, puissamment mis en lumière par une monographie familiale, vient éclairer en retour celui de tous les échevins qui vivent à proximité de contribuables portant le même patronyme.

Il est une autre circonstance où la monographie vient au secours de la prosopographie, c'est lorsqu'il s'agit d'exploiter des sources qui ne se plient pas à la statistique, comme la littérature. Ce genre de monographie n'est pas «familiale » à proprement parler, mais peut être un auxiliaire de choix pour comprendre les familles et des groupes sociaux. C'est tout du moins l'apport de l'étude du Tournoiement des dames de Paris, écrit par Pierre Gencien à la fin du XIII ${ }^{\mathrm{e}}$ siècle. Il s'agit d'un poème drolatique de 1796 vers qui raconte comment ce changeur a assisté, en rêve, à un tournoi extraordinaire mettant aux prises des dames de Paris. C'est une fiction qui met en scène des personnages réels, en l'occurrence de vraies bourgeoises de la capitale, toutes parentes, amies ou connaissances de l'auteur. Il n'y a pas grand chose à tirer du point de vue statistique de cette source: il suffit, pour la rendre pertinente du point de vue de la prosopographie de l'échevinage, de constater que Pierre Gencien est le père d'un échevin et que 29 des 91 femmes qu'il cite appartiennent à des lignages qui ont donné ou qui donneront des magistrats à la municipalité. En revanche, cette source a beaucoup à nous apprendre sur la culture littéraire de son auteur et de son public, puisqu'il s'agit d'une œuvre mondaine destinée à être diffusée - aux antipodes des livres de raison, à usage strictement interne. L'étude minutieuse de la structure, du vocabulaire, des motifs, des références mises en œuvre pour tisser le texte, montre que Pierre Gencien avait une connaissance intime des relations de tournois et du Conte du Graal de Chrétien de Troyes dont il fait un pastiche exact; ses références sont aussi bien classiques (la Chanson de Roland) que récentes (le Roman des sept sages de Rome); il manifeste une réelle habileté littéraire qui suppose une culture bien maîtrisée et du talent; enfin, il montre une parfaite aisance dans l'univers héraldique, puisqu'il ne blasonne pas moins de 26 écus, avec toute la 
science dont on est capable à son époque. Cette maîtrise littéraire, que l'on est obligé de supposer aussi chez le public de Pierre Gencien, tant son poème parodique repose sur une esthétique de la connivence, vient en retour éclairer d'autres œuvres produites par des bourgeois : une lettre de Jean Sarrazin à Nicolas Arrode (la première en français d'un genre appelé à une grande fortune), une chanson courtoise de Philippe Paon, une chronique anonyme qui s'intéresse trop aux Parisiens et aux échevins pour n'être pas produite par un bourgeois, de même qu'une autre chronique qui manifeste des goûts semblables, écrite par un certain Geoffroy, dit « de Paris » parce qu'on ne sait pas qui c'est ${ }^{21}$. Personne ne savait quoi conclure de l'existence de ces œuvres disparates, en dehors du fait que la capitale est un foyer culturel brillant ${ }^{22}$. On peut désormais affirmer que ces textes sont la preuve d'un très bon niveau de culture littéraire, partagé par l'ensemble de ces bourgeois.

La lecture du Tournoiement des dames apprend beaucoup également sur la mentalité de l'auteur et de son public, sur leurs idées, et cet accès à leur conscience est trop rare pour être négligé ! En effet, un bourgeois ne peut pas écrire une relation de tournoi sans poser la question de ses rapports avec la noblesse, tant ce jeu fait partie de la culture aristocratique. Cette question se complique ici de l'ambition affichée d'être comique : qu'y a-t-il de plus drôle que de voir des femmes pratiquer ce sport brutal ? Et que dire, lorsque l'inversion sexuelle de double d'une inversion sociale ? Le problème est au fond de savoir si le rire de Pierre Gencien est subversif ou non, si le comique est simplement parodique ou s'il est satirique, bref si ce changeur cherche à amuser en tournant gentiment en dérision les pratiques sociales de la bourgeoisie parisienne, qui sont effectivement souvent proches de celles des nobles ${ }^{23}$, ou s'il veut dénoncer cette confusion des genres. La réponse se trouve dans la confrontation de cette source littéraire avec les autres, et en particulier les unions matrimoniales, résolument bourgeoises, les lettres d'anoblissement ou la titulature, dont on a vu ce qu'il fallait en penser. Mais on ne comprendrait pas, si le Tournoiement était une satire des goûts bourgeois pour la chevalerie, que le ton soit si louangeur, les femmes si belles, leur mari si riche; on ne comprendrait pas non plus cette volonté de blasonner tous les écus bourgeois au point de faire de ce poème le premier armorial de la bourgeoisie parisienne ; on ne comprendrait pas enfin que le lignage de l'auteur (et son blason!) y soit mis à l'honneur

\footnotetext{
${ }^{21}$ Jean Sarrazin, lettre à Nicolas Arrode, 1249, éd. Lucien Foulet, Paris, 1924 ; Guy Reynaud, Bibliographie des chansonniers français des XIII ${ }^{\mathrm{e}}$ et XIV ${ }^{\mathrm{e}}$ siècles, 1884, réed. Osnabrück, 1971, nº 1286 (Philippe Paon) ; Pierre Gencien, Tournoiement as dames de Paris, éd. par Andrea Pulega, Ludi e spettacoli nel medievo. I tornei di dame, Milan, 1970, p. 21-63 ; Chronique parisienne anonyme, éd. A. Hellot, M.S.H.P., 11, 1884, p. 1-207 ; Geoffroy de Paris, Chronique métrique, éd. Armel Diverrès, Strasbourg, 1956.

${ }^{22}$ Raymond Cazelles, Paris..., p. 118, 393 et suiv.
} 
tout au long des vers... Force est donc de constater que ces bourgeois de Paris rient sans rougir de leurs pratiques sociales aristocratiques, parce qu'ils assument sans complexe leur originalité dans la société de leur temps. Loin de chercher la fusion dans le second ordre, ils aspirent à s'affirmer comme noblesse de la bourgeoisie.

Le renversement du rapport traditionnel entre monographie familiale et prosopographie étant admis comme une nécessité dans le cadre de cette recherche, il reste néanmoins une question gênante: de quelle réalité sociale ce panel de 47 bourgeois est-il représentatif ? Autrement dit, à qui peut-on généraliser les conclusions les concernant ?

\section{Qui représentent les 47 cas du panel ?}

La première certitude, c'est que leur cas peut s'étendre légitimement à tous les échevins parisiens du XIII ${ }^{\mathrm{e}}$ et $\mathrm{XIV}^{\mathrm{e}}$ siècles, avec quelques nuances peut-être pour ceux qui ont vécu après la crise de 1348-1358 ${ }^{24}$. Si les analyses des différentes formes du pouvoir sur la ville n'intéressent que les magistrats municipaux, en revanche les familles échevinales sont assez ouvertes par leurs alliances matrimoniales à d'autres familles bourgeoises pour que l'on puisse généraliser leurs pratiques sociales (structure familiale, habitat, culture, piété, expression de la notabilité) à tous les riches bourgeois de la capitale. Ces analyses peuvent s'appliquer dans une moindre mesure à la petite bourgeoisie (en particulier le mode de gestion du patrimoine, l'architecture de la résidence), en posant l'hypothèse que les citadins moins riches agissent de même, sur un mode mineur.

Toutefois cette étude ne donne pas une vue d'ensemble de toute la bourgeoisie, en encore moins de la population de Paris. Ce travail s'inscrit donc dans le cadre des « monographies de synthèse » qui sont l'étape nécessaire entre les recherches de détail et les histoires générales de Paris. Toutefois il serait vain d'espérer généraliser cette démarche à toute la société, sur le modèle proposé par Raymond Cazelles, en substituant des prosopographies aux monographies familiales, car elles ne peuvent porter que sur l'élite sociale, celle qui donne une prise à l'historien pour reconstituer des généalogies, des patrimoines, des carrières ; or les individus sont presque impossibles à saisir dans la petite bourgeoisie, faute de source - le cas de Geoffroy de Saint-Laurent est une exception qui s'explique par la conservation de son cartulaire dans les archives de Saint-Magloire ${ }^{25}$. Cette aubaine documentaire est selon toute

\footnotetext{
${ }^{23}$ On a vu que les bourgeois de Paris lisaient volontiers des romans de chevalerie, on ajoutera que, comme ceux des autres villes du royaume, ils pratiquent aussi la joute.

${ }^{24}$ Toutefois ni la peste, ni la crise politique ne semblent avoir bouleversé la grande bourgeoisie de Paris, tandis que la crise économique liée aux débuts de la guerre de Cent ans semble de bien faible impact en comparaison de celle du début du $X V^{\mathrm{e}}$ siècle.

${ }^{25}$ Anne Terroine et Lucie Fossier, Un bourgeois...
} 
vraisemblance rarissime et on ne peut compter sur ce type de source pour traiter du cas d'autres bourgeois modestes de la capitale. On en est donc réduit à articuler grands agrégats juridiques et conceptuels élaborés par les hommes du Moyen Âge (bourgeois et forains, métiers jurés et métiers libres, maîtres et valets, gros et menus, etc.) avec des aubaines documentaires, voire des anecdotes. L'étude de la petite bourgeoisie de Paris se distingue cependant de celle des marginaux ${ }^{26}$ en ce sens qu'elle peut placer, entre la coutume et l'anecdote, les analyses statistiques que l'ont peut tirer des rôles d'imposition et des dizaines de milliers de noms qu'on y trouve, avec l'adresse, la taille et parfois la profession du contribuable. Mais ces analyses ne peuvent être fondées que sur une appréhension globale de la source et non sur une série d'analyses de cas, que l'échelle soit individuelle ou familiale. Cela nécessite donc l'invention de méthodes nouvelles ${ }^{27}$.

Pour conclure, sur le fond, on pourrait dire que la prosopographie de l'échevinage a permis d'apercevoir la tête et la colonne vertébrale du mammouth - et c'est déjà heureux - mais que le reste de l'anatomie de la population de la capitale et en particulier de sa bourgeoisie artisanale ne pourra être appréhendé qu'avec d'autres méthodes.

Le détour par la prosopographie peut donc résoudre la contradiction propre à la monographie, non seulement parce qu'elle porte sur un plus grand nombre de cas, mais surtout parce qu'elle est sous-tendue par une hypothèse qui rassemble les cas autour d'un même problème. La prosopographie de l'échevinage n'est pas une simple mise en série de monographies familiales, même si elle a mis en œuvre des méthodes identiques, telles que l'identification patiente des personnes et la reconstitution de tableaux de filiation. En effet, elle ne vise pas à l'exhaustivité, mais seulement à atteindre la masse critique de documents nécessaire pour cerner les profils individuels et familiaux; de même, elle ne cherche pas à étudier le cas pour lui même, dans toute sa singularité, mais vise au contraire à comprendre ce qui le rapproche des autres, pour en faire un portrait de groupe.

Toutefois cette prosopographie s'est voulue limitée quantitativement, ce qui a permis de conjuguer la rigueur de son approche méthodologique avec la profondeur des analyses qualitatives. Les résultats apportés par la monographie familiale prennent alors tout leur sens, mais il fallait d'abord les remettre à leur place, c'est-à-dire insérer le cas dans une trame plus

\footnotetext{
${ }^{26}$ Bronislaw Geremek, Les marginaux...

${ }^{27}$ C'est ce que fait Caroline Bourlet dans sa thèse en cours, Pouvoir, espace et structures sociales à Paris sous les règnes de Philippe le Bel et ses fils, sous la direction de Philippe Braunstein.
} 
vaste. C'est donc à une prosopographie qualitative à laquelle la recherche a abouti, preuve qu'il n'y a pas d'opposition de fond entre les deux méthodes.

On peut même se demander si cette prosopographie ne peut pas être incluse dans la définition de la «monographie familiale», tant le concept est souple et susceptible d'extension infinie : il peut concerner une famille vécue, un lignage partageant le même patronyme, une parentèle cognatique, et pourquoi pas, comme c'est le cas des échevins parisiens, un milieu endogame? La prosopographie de l'échevinage peut apparaître, à cet égard, comme une monographie familiale, à condition que l'on n'oublie pas que le point de départ de la réflexion était institutionnel et individuel, plutôt que familial, et que cette réflexion était sous-tendue par un problème, celui du mode de domination et des pratiques sociales propres au patriciat.

Boris Bove

Université de Paris 8 
Tableau 1 - Evolution de la famille de Pierre III Marcel [94]

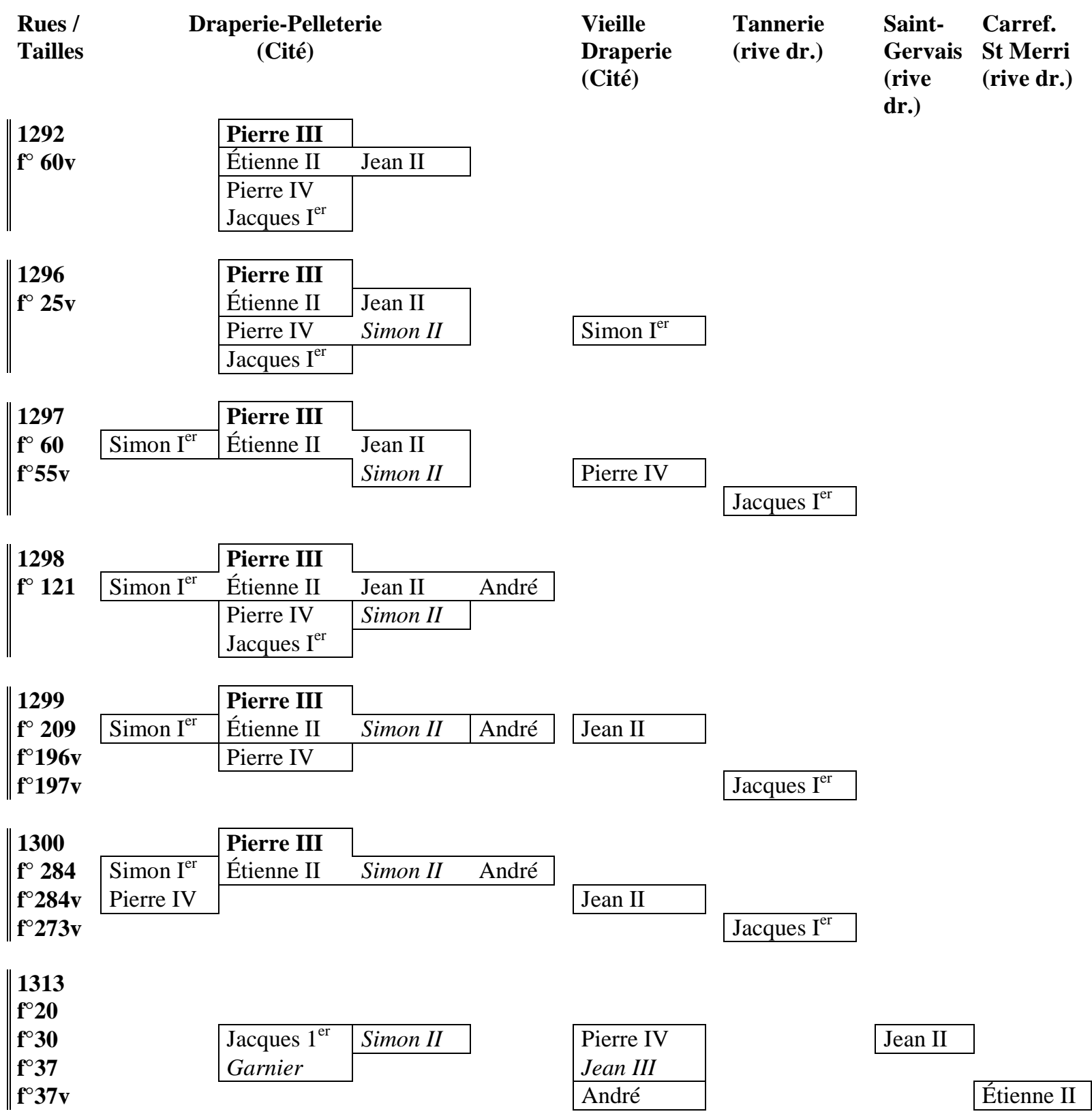

En gras : le père / en maigre : les fils / en italique : les petits-fils 


\section{Légende du graphique}

1. GIFFART Philippe [48

2. GENCIEN Jean [98]

3. PISDOE Guillaume [60]

4. BOURDON Guillaume [14]

5. BOUCEL Baudoin [78]

6. BOURDON Jacques [15]

7. DE RESSONS Guillaume [95]

8. DE REIMS Thierry [69]

9. BOUCEL Guillaume [79]

10. PISDOE Eudes [59]

11. BARBETTE Jean [6]

12. PISDOE Renaud [63]

13. LA PIE Jean [50]

14. DES NEFS Henri [45]

15. BOURDON Etienne [12]

16. BOURDON Adam [11]

17. BOUCEL Adam [10]

18. MARCEL Gilles [56]

19. AMI Guillaume [1]

20. DE VALENCIENNES Evroin [44]

21. PACY Raoul [38]

22. LE FLAMENT Nicolas [53]

23. DE RUEIL Jean [40]

24. ARRODE Nicolas [3]

25. DE TREMBLAY Garnier [43]

26. BIGUE Jean [9]

27. BOURDON Simon [18]

28. BOURDON Guillaume [13]

29. BARBETTE Jean [7]

30. SARRAZIN Jean [68]

31. PISDOE Guillaume [64]

32. BOURDON Pierre [16]

33. BELOT Jean [8]

34. DE RUEIL Jean [39]

35. FLEURY Jean [31]

36. DE ST BENOIT Thomas [42]

37. DU CELIER Jean [90]

38. TOUSSAC Guillaume [71]

39. POPIN Jean [66]

40. PACY Raoul [37]

41. PAON Adam [58]

42. MARCEL Garnier [55]

43. AUGIER Jean [4]

44. BOURDON Renier [17]

45. TRISTAN Gencien [72]

46. PISDOE Jean [62]

47. MARCEL Etienne [54]

48. LE FLAMENT Geoffroy [51]

49. DE ST BENOIT Simon [41]

50. MARCEL Pierre [94]

51. PACY Nicolas [36]

52. DE DAMMARTIN Geoffroy [29]

53. PISDOE Guillaume [61]

54. ARRODE Jean [2]

55. SARRAZIN Jean [67]

56. GENCIEN Jean [47]

57. BARBETTE Etienne [5]

58. FLEURY Geoffroy [30]

59. HAUDRI Etienne [49]

60. POILEVILAIN Jean [65]

61. PACY Jean [35] 